\title{
Survival and cancer risk in an unselected and complete idiopathic inflammatory myopathy cohort from south-east Norway.
}

Cecilie Dobloug ${ }^{1}$, Torhild Garen ${ }^{1}$, Jan Tore Gran ${ }^{1}$, Cathrine Brunborg ${ }^{2}$ Øyvind Molberg ${ }^{1,3}$

'Department of Rheumatology, Oslo University Hospital (OUH), Oslo, Norway,

${ }^{2}$ Department of Biostatistics, Epidemiology and Health Economics, OUH, Oslo, Norway

${ }^{3}$ Institute of Clinical Medicine, University of Oslo, Oslo, Norway

Corresponding author:

G. Cecilie Dobloug, Department of Rheumatology, Oslo University Hospital, Pb 4950,

Nydalen, N-0424 Oslo, Norway

Phone; 0047-23074878, mail; gcdobloug@doctors.org.uk or gdobloug@,ous-hf.no

Short running title; Idiopathic inflammatory myopathies- survival and cancer risk. 


\begin{abstract}
Objective; To perform mortality and cancer analyses across unselected and complete cohorts of patients with Idiopathic Inflammatory Myopathy (IIM) resident in South-east Norway (denominator population 2.6 million), between 2003-12.
\end{abstract}

Method: IIM cases were identified by comprehensive searches through patient administrative databases followed by manual chart review. Polymyositis (PM) and Dermatomyositis (DM) cases were classified by the Peter and Bohan and/or Targoff diagnostic criteria and sporadic Inclusion Body Myositis (sIBM) by the European Neuro-Muscular Centre (ENMC) criteria from 1997 and/or 2011. Every patient was matched for sex, age and residential area with 15 controls without IIM, drawn from the national population registry.

Results: Total mortality in the IIM study cohort was 27\% (87/326). Standardized mortality rate (SMR) was higher in DM (2.6) than PM (2.4) and SIBM (1.7). IIM related death causes were frequent (64\%) and included cancer (all IIM subsets), aspiration (sIBM), pulmonary complications (PM/DM) and infections (PM/DM). Mortality risk factors were older age at diagnosis and cancer (all IIM subsets), and reduced lung function (PM/DM). Cancer risk was increased in DM (Standard Incidence Rate 2.0) and PM (SIR 1.3), but not in SIBM (SIR 0.9). Ovarian cancer $(8.3 \%)$ and lung cancer $(5.5 \%)$ was more prevalent in DM than in the general population (1.1\% and $2.9 \%$, respectively).

Conclusion: This population-based study, with individual IIM case assignment and matched unexposed controls, shows that mortality rates and cancer risk remain elevated in DM as well as PM. SMR was also increased in sIBM, but several deaths in this group were due to potentially preventable causes. 


\section{INTRODUCTION}

Idiopathic inflammatory myopathies (IIM) are chronic, systemic disorders of unknown aetiology. They are defined by progressive loss of striated muscle tissue and include three major clinical syndromes; dermatomyositis (DM), polymyositis (PM) and sporadic inclusion body myositis (sIBM).[1-5] PM and DM cause symmetric, proximal weakness, sIBM on the other hand, is more asymmetric and involves the quadriceps and finger flexors.[6-8] Myositis associated auto-antibodies (MAA) are frequent, particularly in PM and DM [9] and seem promising as markers for clinical subtypes [10-12] and cancer-associated myositis.[9, 13]

New classification criteria for the entire IIM group are in progress [14], but to date DM and PM are usually classified by the 1975 Peter and Bohan diagnostic criteria, $[15,16]$ or the revised 1997 criteria suggested by Targoff et al. [17] Classification of sIBM is mostly by the 1995 Griggs pathology criteria[18] or the more clinically based European Neuromuscular Centre (ENMC) criteria, first launched in 1997 [19] and last updated in 2011 as the ENMC IBM Research Diagnostic criteria.[20]

Studies from the 1970s indicated increased mortality in PM/DM. [21-25] Later work have confirmed this, but shown large variation in mortality rates, probably due to differences in patient selection, classification, loss to follow-up and treatment related issues.[4, 5] Peter and Bohan criteria have been applied in eight studies on mortality [3, 4, 26-31] but only one of these eight studies was performed on an unselected population based cohort. [3] This study, which is the largest mortality study to date, performed hospital discharge searches for $\mathrm{PM} / \mathrm{DM}$ cases by the $7^{\text {th }}$ International Classification of Disease version 7 (ICD-7) codes and subsequent case assignment by chart review to identify $248 \mathrm{PM} / \mathrm{DM}$ patients diagnosed in Finland from 1969-1985, followed until death or 1995. Mortality rates in this study were assessed by life-tables [3], and not by matched population controls [28]. Available data 
indicate that major causes of death in PM/DM are cancer, infections and cardiopulmonary complications. [3, 5] Prognostic factors vary between studies, but older age at diagnosis, cardiopulmonary disease, cancer and the presence of MAA have all been associated with poor outcome.[5, 32-34]

Knowledge on survival and mortality in sIBM is more limited. Long-term follow-up studies from Europe have not found reduced life expectancy.[6] In contrast, a recent multinational study on selected patients, with no detailed case definition data available, reported increased mortality, with an estimated Standardized Mortality Ratio (SMR) of 6.58 for patients aged $41+$, and 4.82 for patients over $70 .[34]$

Associations between PM/DM and solid tumours have been shown in populationbased studies with different case assignment strategies. Overall, these studies observed a 2-to 4 fold increased cancer risk in PM/DM, with highest incidence rates in the DM subset and in males older than 50 years.[32, 35-38] Cancer diagnosis can precede, parallel, or follow $\mathrm{DM} / \mathrm{PM}$ diagnosis. The largest cancer study in PM/DM to date was a pooled analysis of data from Sweden, Denmark, and Finland.[35-37] The study included 618 DM and 914 PM patients identified by ICD-7 or ICD- 8 hospital discharge diagnoses, but less than $1 / 3$ of these cases had their PM/DM diagnosis verified by chart review[32]. Standardized Incidence Rates (SIR) were estimated using National cancer registry data as reference and found to be 3.0 in DM and modestly increased, at 1.3 in PM.[32]. A recent retrospective study on biopsy-proven IIM cases from Victoria, Australia reported increased cancer risk across all IIM subsets, including sIBM. [37] Cancer subtype analyses indicated that the most common PM/DM associated malignancies were of ovarian, lung, gastrointestinal, breast or haematological origin.[32, 35-38]

Data on the survival and cancer risk across all the IIM diagnoses in the general population are rather limited. There are few studies based on unselected groups and few 
undertaken on sIBM. In the current study, based on sound case definitions, we assess SMR, cancer risk in an unselected IIM cohort that includes all PM, DM and sIBM patients resident in South-East Norway (with a denominator population of 2.64 million) between January 2003 and December 2012. All identified IIM patients were matched with15 unexposed controls matched for age, sex and residential area from the national population registry. Cox regression was performed in IIM group and utilized to identify risk factors for mortality in all the three disease groups. 


\section{MATERIALS \& METHODS}

\section{Study cohort}

South-east Norway consist of 10 counties with 2642246 inhabitants (by December $31^{\text {st }}, 2012$ ), includes the largest cities in Norway and consists mostly of urban/suburban dwellings. The IIM cohorts were selected from this denominator population and consisted of every person who fulfilled the study inclusion criteria for PM/DM or sIBM (see below).

There are 10 public hospitals in South-east Norway; the largest is Oslo University Hospital (OUH), which is the primary local hospital for Oslo (with 600000 inhabitants) and referral hospital for all the 10 counties in the region. In Norway, patients with IIM are followed by specialists at the public hospitals; PM and DM predominantly by rheumatologists[39], while sIBM patients are followed either by neurologists or by rheumatologists.[40] Since 1999, all patients contacts in the specialist health service were electronically registered by $10^{\text {th }}$ revised version of the International Classification of Disease (ICD-10) codes.

\section{Study inclusion criteria}

Patients were included if they fulfilled the following criteria: (A) Disease classifiable as adult PM or DM by the Peter \& Bohan and/or the Targoff criteria, or as sIBM by the 1997 ENMC sIBM criteria and /or the 2011 ENMC IBM Research Diagnostic Criteria. (B) Age above 18 years. (C) Patient registered in the Norwegian Central Population Register with a home address in South-East Norway between January $1^{\text {st }} 2003$ and December $31^{\text {st }} 2012$.

\section{Case finding strategy}

This has previously been described in detail.[39, 40] Briefly, two major acquisition routes were utilized to identify every adult patient with PM/DM and SIBM living in South- 
east Norway between 2003 and 2012; (1) broad ICD-10 based searches across the patient administrative databases at all of the public hospitals in the study area followed by manual chart review of all potential IIM cases, and (2) retrospective review of all muscle biopsy reports encoded with inflammation by a neuropathologist. This muscle biopsy report review was performed to identify all potential sIBM patients and aid the process of differentiating sIBM cases from cases with PM. Each patient was finally discussed on a one to one notion and assessed together with the clinical information gathered over a 10 year period.

\section{Disease characteristics:}

The time of symptom onset was recorded. Disease duration was defined as the time from diagnosis to the end of the observation period (at study end $31^{\text {st }}$ of December 2012, or at the time of death). Time of death and cause of death was noted when applicable. Clinical, laboratory and radiological parameters were recorded at disease onset and cumulatively during follow-up as described [39, 40];

\section{Assessment of Standard mortality rate (SMR) and survival rates:}

All residents of Norway have a unique national registration number that allows complete follow-up until death or migration. This personal identification number system allows for case-controlled mortality studies. Hence, every patient was matched with 15 controls without IIM, drawn from the National Population Registry via Statistics Norway. The matching included gender, year and month of birth and area of residence. Vital status at January 1st, 2013, was provided for patients and controls by the national population registry. SMR and survival rates were calculated using data from the matched controls.

\section{Causes and risk of dying.}


Causes of death were identified by manual chart review of medical charts, death certificates and autopsy and from ICD-10 codes from the official Cause of Death Registry in Norway. When there was discrepancy between information, data from the medical charts were used. The cause of death in the control group was given by Statistics Norway. In the IIM group causes of death was classified as either as IIM related mortality or mortality unrelated to IIM. Individual cases were discussed on a case to case notion amongst the authors when in doubt about the relation to IIM. If there was no notification of the death-it was named unknown. When assessing risk factors for death, we utilized Cox regression on the IIM patients, to identify clinical parameters associated with increased hazards.

\section{Cancer}

The Cancer Registry of Norway provided data on cancer occurrence between 20032012 in the IIM patients and their matching controls, coupled via their personal identification numbers. Time and place of cancer diagnosis, stage, type and metastasis, organ of origin, and if deaths were related to cancer, was noted. IIM patients diagnosed with cancer before 2003 or after 2012 were identified during by chart review and pathology reports, and were included to assess if the cancer occurred +/-3 years prior or after IIM diagnosis. Due to the rarity of individual cancer subtypes, life tables from the Cancer Registry of Norway were utilized for comparative subtype frequency analyses.

\section{Ethical aspects}

The Regional Committee of Medical ethics in Southern Norway (REK Sør), the Norwegian Ministry of Health (the Norwegian Patient Registry) and Privacy Policy Department at OUH have all approved this study with all aspects related to patient data recording and ethical aspects related to the handling of patient sensitive material.

\section{Statistical analysis}


Statistical analysis was undertaken by SPSS, version 20/21 and STATA[41].

Descriptive statistics; continuous variables with normal distribution were presented as mean with Standard Deviation (SD) or 95\% Confidence Interval (CI). Categorical variables were presented as numbers and percentages. Pearson's Exact test and Chi Squared test was utilized for the comparison of independent groups of categorical data, significance level was $p<0.05$. Cumulative survival rates were calculated by the Kaplan-Meier method and significance and equality of survival distribution between controls and patients for the different levels of diagnosis was tested with the log-rank test (Mantel Cox). Survival curves were used to compare with the curves of the controls. Hazard rates were calculated by usage of SPSS Cox regression, hazard rates, the numbers presented in tables were Wald and significance level, $\mathrm{p}<0.05$ was interpreted as significant. All variables were tested separately on an individual basis by Cox regression to assure that the level of significance not was affected by multiple testing. [41]

STATA was utilized when calculating SMR (Standard Mortality Rate) and SIR (Standard Incidence Rate), and Incidence rate ratio, these numbers were given with a 95\% CI. . Mean time to death in years and Mean time from cancer to death measured in years were calculated utilising SPSS. STATA was also used when calculating 2,5 and 10 year survival in patients and controls.[41] 


\section{RESULTS}

\section{IIM patient cohorts}

As previously described $[39,40]$, the searches through the hospital databases identified 3160 patients who had received ICD-10 codes potentially compatible with IIM. These 3160 patients partly overlapped with the 500 patients who had muscle biopsies encoded with inflammation. Detailed, retrospective review of chart and histology data showed that only 326 of the patients screened were classifiable as IIM; 226 patients met the Targoff diagnostic criteria for PM/DM (98 PM, 128 DM) and 208 patients met the Peter \& Bohan criteria. The remaining 100 patients (40 female and 60 male) met the 1997 and/or 2011 ENMC diagnostic criteria for sIBM.[40] The 1997 criteria were met by 92 patients, and the 2011 criteria were met by 95 patients.[40] The sIBM patients were older at diagnosis (mean age 66.9 years), had longer diagnostic delay (5.6 years), but shorter total disease duration (5.5 years) than PM and DM patients (Table 1). By the end of the study period, 87/326 patients had died (Table 1). Mean time from diagnosis to death was shorter in DM (4.2 years) than in PM and sIBM (Table 1).

\section{Mortality}

Highest SMR (2.6) was observed in DM (Table 1). Stratification by time of diagnosis showed that SMR was significantly lower in DM patients diagnosed before 2003 (SMR 1.3) than in patients diagnosed from 2003 and onwards (SMR 3.2) (Table 1). The same trend was seen amongst the PM patients with SMR 1.9 in patients diagnosed before 2003 and 2.9 in patients diagnosed after 2003 (Table 1). In sIBM, who had a lower total SMR (1.7), the differences were less pronounced.

\section{Survival rates}


Overall, the survival in the IIM patients was lower than in the matched controls at all time-points. This was verified by test of equality of survival distributions between controls and patients by Log Rank (Mantel Cox) $(\mathrm{p}<0.000)$ (Figure 1). The cumulative two year survival for the whole IIM group was $87 \%$, compared to $96 \%$ for age and gender matched controls (data not shown). Survival rates at two years were comparable across the three IIM; DM $86 \%$, PM $87 \%$ and sIBM 87\% (Figure 1). Similar overall survival rates were also observed after five years disease duration; DM 77\%, PM 77\% and sIBM 78\% (Figure 1). Larger differences were seen at 10 years, where sIBM appeared to have a poor outcome with $42 \%$ cumulative survival, but the corresponding low survival in the matched controls show that this was an effect of old age at diagnosis (Figure 1c). DM patients had a $55 \% 10$ year survival and the lowest age at diagnosis compared to $78 \%$ in their matching controls (Figure 1b), compared to PM with 60\% 10 year survival and $82 \%$ in controls (Figure 1a).

\section{Causes of death}

Causes of death were related to IIM in 56/87 (64\%) patients (Table 2). Myositis associated cancer was defined as occurring +/-3 years from the IIM diagnosis. In DM, nine patients died secondary to myositis-associated cancer compared to five and three in PM and sIBM, respectively (Table 2). Interstitial lung disease (ILD)/Pulmonary Hypertension (PH) caused nine deaths in the DM group compared to none in the sIBM group and five in the PM group. Ten patients died of infection and in some of these cases, the infection was probably treatment related, although not well documented as such in journals. Aspiration associated deaths were mostly seen in sIBM (seven cases). Among the death causes that were not related to IIM, myocardial infarction/heart failure (14\%) and cancer $(8 \%)$ were the most common (Table 2). 


\section{Mortality risk factors}

Mean age at diagnosis was associated with increased mortality across all three IIM subsets, with an increase in risk of 10\% in PM/DM and 20\% in sIBM for every increase in year $(\mathrm{P}=0.000)$. Disease duration was protective in all patients $(\mathrm{P}=0.000)$, with a lower risk in patients surviving past the first years of diagnosis (Table 3). Cancer was identified as a risk factor in all IIM subsets, with hazard rates $(\mathrm{HR})$ of $9.0(\mathrm{P}=0.000)$ in $\mathrm{DM}, 2.7$ in $\mathrm{PM}(\mathrm{P}=0.013)$ and 2.5 in $\operatorname{sIBM}(\mathrm{P}=0.025)$ (Table 3$)$. Diffusing capacity for carbon monoxide (DLCO) $<60 \%$ (a marker for pulmonary involvement) was associated with a 13 fold risk in PM patients and a 5 fold risk in $\mathrm{DM}$ patients $(\mathrm{P}=0.002$ and $\mathrm{P}=0.018$ respectively). In $\mathrm{DM}, \mathrm{FVC}<70 \%$ was also associated, with HR 7.0 (Table 3). The frequency of anti-Jo-1 was very high in our cohort (Table 1), but neither anti-Jo-1, nor MAA in total were associated with increased HR (Table 3). All variables were in addition tested individually, to overcome the possibility of multiple testing influencing the level of significance.

\section{Cancer}

Total frequency of cancer in the IIM cohort was $24 \%$ (78/326), compared to $16 \%$ $(800 / 4889)$ in the age- and gender matched controls. Absolute and relative cancer frequencies differed between IIM subtypes; it was 24\% (31/128) in DM and 11\%(215/1926) in DM controls, 24\% (23/98) in PM and 17\% (245/1456) in PM controls and finally, 24\% (24/100) in sIBM and 23\% (340/1507) in the matched sIBM controls. Hence, the standard Incidence ratio (SIR) for cancer was higher in DM (2.0) than in PM (1.3) and SIBM (1.0) (Table 4). Myositis associated cancers, defined as occurring +/- 3 years of diagnosis, were seen in $18 \mathrm{DM}$ patients, $11 \mathrm{PM}$ patients, and $10 \mathrm{sIBM}$ patients (Figure 2). Mean time from diagnosis to cancer was -2.4 years in PM, -3.1 years in DM and 2.9 years in sIBM (Table 4). Mean time 
from cancer to death was lowest in DM patients 4.2 years compared to 6.9 and 6.1 years in PM and sIBM respectively.

\section{Cancer types}

Cancer types and frequencies differed between the disease groups. The most frequent encountered cancers in DM were lung and ovarian cancer (Table 4). Life table data from the Norwegian Cancer Registry showed that the 10-year accumulated, age-adjusted frequency of ovarian cancer in females from the general population was $1.1 \%$; lower than the $8.3 \%$ frequency observed in the female DM patients. According to the same life tables, accumulated, age-adjusted frequency of lung cancer was 3.1\%, compared to $5.5 \%$ in DM. In PM, haematological malignancies were the most common cancer types (Table 4). Ageadjusted frequency of haematological malignancies in life tables between 2003 and 2012 were $2.9 \%$, compared to $4.1 \%$ in the PM patients. The most common cancer types observed in sIBM were prostate, colorectal cancers and haematological malignancies (Table 4), and this frequency distribution was the same as in age-adjusted life table data (data not shown). 


\section{DISCUSSION}

Cancer risk and mortality rates are important topics in IIM, but have rarely been assessed in population based settings. Here, these issues were approached by a case controlled study design with cases from unselected PM, DM and sIBM cohorts. Our results give further support to the notions that malignancy, particularly of ovarian and lung origin, and ILD are major determinants of mortality in DM, and that PM is also associated with increased, albeit more moderate, mortality and cancer risk. The results also show, for the first time that unselected sIBM has slightly increased SMR, but no signal for cancer.

We believe that the current study has major strengths. Firstly, the case assignment strategy applied was robust and captured (nearly) all IIM patients resident in the study area over a 10 -year period. Secondly, due to the unique identification numbers and official mortality statistics in Norway, none of the patients identified were lost to follow-up. Thirdly, mortality and cancer data captured by manual chart review was confirmed and extended by data sets from the Norwegian Cancer and Mortality Registries. Finally, the use of age and sexmatched unexposed controls, instead of life-tables, gave us a unique opportunity to directly compare data across all the mortality and cancer analyses.

The weakness of this study was its retrospective nature of data-collection. All data acquisition on causes of death and clinical information were based on medical records and death certificates with potential missing information. Another limitation, which we were aware of, and aimed to quantify, was immortality bias. This was particularly evident in DM, where patients diagnosed prior to 2003 had much lower SMR than those diagnosed from 2003 and onwards.

At $55 \%$ and $60 \%$ respectively, the 10 years cumulative survival rates in our population based DM and PM cohorts were no higher than previously observed in single-centre cohorts, 
$[5,27,33]$. The 10 year survival in sIBM was even lower $(42 \%)$, but the low survival in the matched controls (57\%) showed that this was an effect of age. The age effect was also apparent in the risk factor analyses where mean age at diagnosis stood out as an important determinant of mortality across all the IIM cohorts. Interestingly, we observed that disease duration was protective, with lower mortality in patients surviving the first five disease years.

The mortality risk analyses showed increased HR for cancer in all the IIM subsets. As in previous studies, the risk was highest in $\operatorname{DM}[5,33,42,43]$. Reduced pulmonary function, expressed by $\mathrm{FVC}<70 \%$ was also associated with mortality in DM, but not in PM. DLCO $<60 \%$, on the other hand, had a higher HR in PM than in DM. Taken together, these data appear in line with a study on $107 \mathrm{PM} / \mathrm{DM}$ patients where reduced FVC and DLCO was associated with mortality.[44]

Several previous studies have reported decreased survival in PM/DM patients with MAA $[5,13,29,45-47]$. Here, we did not find that MAA was associated with mortality. We speculate that this may be an effect of differential patient selection. Our PM/DM cases were unselected, while the previous studies were from specialized centres that possibly selects for $\mathrm{PM} / \mathrm{DM}$ patients with more severe disease and higher frequency of MAA-related organ complications like ILD and $\mathrm{PH}$. Another possibility is that the recent availability of more efficient treatment has improved the relative survival of MAA positive patient subsets.

The major causes of death in PM/DM were cancer, infections (some treatment related, but unfortunately not well documented), and respiratory failure due to ILD and/or secondary $\mathrm{PH}$, as reported by others.[4, 5, 42, 48] Interestingly, in sIBM, aspiration due to dysphagia, a sometimes preventable complication, appeared to be the most important cause of death.

The current study is the first to assess cancer in IIM cohorts based on individual case assignment and with matched controls. Reassuringly, the cancer frequencies, subtype 
distribution and cancer risk factors observed were comparable to previous publications.[5, 32, 36]Moreover, we believe that our data strengthen the notion of a para-neoplastic process in DM-patients, as cancer incidence in this subset was highest within the first year of diagnosis. [35] Notably, it is possible that the concept of cancer associated myositis, based on a window around the time of diagnosis, may be less appropriate in SIBM than in PM/DM, due to the long diagnostic delay ( 5.6 years) seen in these patients.

In conclusion, this is the first study that concurrently have assessed mortality rates and cancer risk across well-defined, unselected IIM cohorts from the same catchment area, and the first to utilize matched controls rather than life tables. The results show that mortality in PM and DM remains high, and provide new insights on survival and risk for malignancies in sIBM. Based on the data we believe that it is reasonable to suggest mandatory cancer surveillance and ILD screening, irrespective of autoantibody status, in DM and PM. Mapping of oesophagus dysmotility for prevention of aspiration should be a priority in sIBM, with the aim of reducing disease related deaths. 


\section{ACKNOWLEDGEMENTS}

The authors thank: -Ellen Ann Antal, Dept. of Pathology, Oslo University Hospital (OUH); Line Sveberg, Dept. of Neurology, OUH; Helle Bitter, Dept. of Rheumatology, Sørlandet Hospital, Kristiansand. Johan Stjärne, Dept. of Rheumatology, Betanien Hospital, Skien; Lars Grøvle, Dept. of Rheumatology, Sykehuset Østfold, Moss; Cecilie Kaufmann and Åse Lexberg, Department of Rheumatology, Buskerud Hospital, Vestre Viken; Olav Bjørneboe, Department of Rheumatology, Martina Hansens Hospital, Bærum Norway, Patrick Stolt, Department of Rheumatology, Innlandet Hospital, Kongsvinger, Knut Mikkelsen, Department of Rheumatology, Innlandet Hospital, Lillehammer, Christian Gulseth, Department of Rheumatology, Betanien Hospital, Telemark, Anne Noraas Bendvold, Department of Rheumatology, Sørlandet Hospital, Kristiansand, Østfold.Tormod Fladby, Department of Neurology, Ahus Hospital, Akershus, Remo Gerdts, Department of Neurology, Vestfold Hospital, Vestfold, Sharka Øygaarden, Telemark Hospital, Telemark, Grethe Kleveland, Department of Neurology, Innlandet Hospital, Lillehammer; Anne-Kathrine Palacios Department of Neurology, Østfold Hospital, Østfold, for providing access to data.

\section{Funding:}

This work has been supported by grants from the Norwegian Women`s Public Health Association and Extrastiftelsen.

Disclosure statement: Competing interest; none declared. 
Author contributions

Conception and design: $\mathrm{CD}, \varnothing \mathrm{M}, \mathrm{JTG}, \mathrm{CB}$

Provision of study materials or patients: $\mathrm{CD}, \varnothing \mathrm{M}$

Collection and assembly of data: $\mathrm{CD}, \varnothing \mathrm{M}$

Analysis and interpretation of the data: CD,ØM,JTG,TG

Drafting of the article: $\mathrm{CD}, \varnothing \mathrm{M}, \mathrm{JTG}, \mathrm{TG}, \mathrm{CB}$

Final approval of the article: $\mathrm{CD}, \varnothing \mathrm{M}, \mathrm{JTG}, \mathrm{TG} . \mathrm{CB}$ 


\section{References}

1. Dalakas MC, Hohlfeld R: Polymyositis and dermatomyositis. Lancet 2003, 362(9388):971982.

2. Christopher-Stine L, Plotz PH: Adult inflammatory myopathies. Best practice \& research Clinical rheumatology 2004, 18(3):331-344.

3. Airio A, Kautiainen $\mathrm{H}$, Hakala M: Prognosis and mortality of polymyositis and dermatomyositis patients. Clinical rheumatology 2006, 25(2):234-239.

4. Marie I, Hachulla E, Hatron PY, Et.al: Polymyositis and dermatomyositis: short term and longterm outcome, and predictive factors of prognosis. The Journal of rheumatology 2001, 28(10):2230-2237.

5. Marie I: Morbidity and mortality in adult polymyositis and dermatomyositis. Current rheumatology reports 2012, 14(3):275-285.

6. Cox FM, Titulaer MJ, Sont JK, Et.al: A 12-year follow-up in sporadic inclusion body myositis: an end stage with major disabilities. Brain : a journal of neurology 2011, 134(Pt 11):31673175.

7. Benveniste O, Guiguet M, Freebody J, Et.al: Long-term observational study of sporadic inclusion body myositis. Brain : a journal of neurology 2011, 134(Pt 11):3176-3184.

8. Cox FM, Verschuuren JJ, Verbist BM, Et.al: Detecting dysphagia in inclusion body myositis. Journal of neurology 2009, 256(12):2009-2013.

9. Tansley S, Gunawardena H: The Evolving Spectrum of Polymyositis and DermatomyositisMoving Towards Clinicoserological Syndromes: A Critical Review. Clinical reviews in allergy \& immunology 2013.

10. Love LA, Leff RL, Fraser DD, Et.al: A new approach to the classification of idiopathic inflammatory myopathy: myositis-specific autoantibodies define useful homogeneous patient groups. Medicine 1991, 70(6):360-374.

11. O'Hanlon TP, Miller FW: Genetic risk and protective factors for the idiopathic inflammatory myopathies. Current rheumatology reports 2009, 11(4):287-294.

12. Brouwer R, Hengstman GJ, Vree Egberts W, Et.al: Autoantibody profiles in the sera of European patients with myositis. Annals of the rheumatic diseases 2001, 60(2):116-123.

13. Chinoy $\mathrm{H}$, Fertig $\mathrm{N}$, Oddis $\mathrm{CV}$, Et.al: The diagnostic utility of myositis autoantibody testing for predicting the risk of cancer-associated myositis. Annals of the rheumatic diseases 2007, 66(10):1345-1349.

14. Tjarnlund. A BM, Rider. L.G, et al: Progress report on the development of new classification criteria for adult and juvenile idiopathic inflammatory myopathies. Annals of the Rheumatic Diseases, The EULAR Journal June 2013, 72(Supplement 3):60/OP0035.

15. Bohan A, Peter JB: Polymyositis and dermatomyositis (first of two parts). The New England journal of medicine 1975, 292(7):344-347.

16. Bohan A, Peter JB: Polymyositis and dermatomyositis (second of two parts). The New England journal of medicine 1975, 292(8):403-407.

17. Targoff IN, Miller FW, Medsger TA, Jr., Et.al: Classification criteria for the idiopathic inflammatory myopathies. Current opinion in rheumatology 1997, 9(6):527-535.

18. Griggs RC AV, DiMauro S et.al: Inclusion body myositis and myopathies. Annals of neurology 1995, Nov;38(5):705-713.

19. Verschuuren JJ BU, Van Engelen BGM, Et.al Inclusion body myositis. London: Royal Society of Medicine Press; 1997.

20. Rose MR: 188th ENMC International Workshop: Inclusion Body Myositis, 2-4 December 2011, Naarden, The Netherlands. Neuromuscular disorders : NMD 2013, 23(12):1044-1055.

21. Rose AL, Walton JN: Polymyositis: a survey of 89 cases with particular reference to treatment and prognosis. Brain : a journal of neurology 1966, 89(4):747-768.

22. Carpenter JR, Bunch TW, Engel A, Et.al: Survival in polymyositis: corticosteroids and risk factors. The Journal of rheumatology 1977, 4(2):207-214. 
23. Medsger TA, Jr., Robinson H, Masi AT: Factors affecting survivorship in polymyositis. A lifetable study of 124 patients. Arthritis and rheumatism 1971, 14(2):249-258.

24. Maugars YM, Berthelot JM, Abbas AA, Et.al: Long-term prognosis of 69 patients with dermatomyositis or polymyositis. Clinical and experimental rheumatology 1996, 14(3):263274.

25. Hochberg MC, Feldman D, Stevens MB: Adult onset polymyositis/dermatomyositis: an analysis of clinical and laboratory features and survival in 76 patients with a review of the literature. Seminars in arthritis and rheumatism 1986, 15(3):168-178.

26. Sultan SM, loannou Y, Moss K, Et.al: Outcome in patients with idiopathic inflammatory myositis: morbidity and mortality. Rheumatology (Oxford) 2002, 41(1):22-26.

27. Schiopu E, Phillips K, MacDonald PM, Et.al: Predictors of survival in a cohort of patients with polymyositis and dermatomyositis: effect of corticosteroids, methotrexate and azathioprine. Arthritis research \& therapy 2012, 14(1):R22.

28. Bronner IM, van der Meulen MF, de Visser M, Et.al: Long-term outcome in polymyositis and dermatomyositis. Annals of the rheumatic diseases 2006, 65(11):1456-1461.

29. Danko K, Ponyi A, Constantin T, Et.al: Long-term survival of patients with idiopathic inflammatory myopathies according to clinical features: a longitudinal study of 162 cases. Medicine 2004, 83(1):35-42.

30. Yamasaki Y, Yamada H, Ohkubo M, Et.al: Longterm survival and associated risk factors in patients with adult-onset idiopathic inflammatory myopathies and amyopathic dermatomyositis: experience in a single institute in Japan. The Journal of rheumatology 2011, 38(8):1636-1643.

31. Torres C, Belmonte R, Carmona L, Et.al: Survival, mortality and causes of death in inflammatory myopathies. Autoimmunity 2006, 39(3):205-215.

32. Hill CL, Zhang $\mathrm{Y}$, Sigurgeirsson B, Et.al: Frequency of specific cancer types in dermatomyositis and polymyositis: a population-based study. Lancet 2001, 357(9250):96100.

33. Yu KH, Wu YJ, Kuo CF, Et.al: Survival analysis of patients with dermatomyositis and polymyositis: analysis of 192 Chinese cases. Clinical rheumatology 2011, 30(12):1595-1601.

34. Barghout VP, M. Benveniste, O. Et.al: Assessment of Mortality in Sporadic Inclusion Body Myositis; a Delphi Panel Technique (P1.025). General Neurology 2014, 82(10):Supplement P1.025.

35. Ungprasert $\mathrm{P}$, Bethina NK, Jones $\mathrm{CH}$ : Malignancy and Idiopathic Inflammatory Myopathies. North American journal of medical sciences 2013, 5(10):569-572.

36. Sigurgeirsson $B$, Lindelof $B$, Edhag $O$, et.al: Risk of cancer in patients with dermatomyositis or polymyositis. A population-based study. The New England journal of medicine 1992, 326(6):363-367.

37. Buchbinder R, Forbes A, Hall S, Et.al: Incidence of malignant disease in biopsy-proven inflammatory myopathy. A population-based cohort study. Annals of internal medicine 2001, 134(12):1087-1095.

38. Madan V, Chinoy H, Griffiths CE, Et.al: Defining cancer risk in dermatomyositis. Part I. Clinical and experimental dermatology 2009, 34(4):451-455.

39. Dobloug C, Garen T, Molberg O, Et.al: Prevalence and clinical characteristics of adult polymyositis and dermatomyositis; data from a large and unselected Norwegian cohort. Annals of the rheumatic diseases 2014.

40. Dobloug C, Antal E, Sveberg L, Et.al: High prevalence of Inclusion Body Myositis in Norway; a population based clinical epidemiology study. European Journal of Neurology 2014.

41. Rothman KJ: Epidemiology, An Introduction, vol. 2nd edition. New York: Oxford University Press; 2012.

42. Marie I, Josse S, Hatron PY, Et.al: Interstitial lung disease in anti-Jo-1 patients with antisynthetase syndrome. Arthritis care \& research 2013, 65(5):800-808. 
43. Marie I, Josse S, Decaux O, Et.al: Comparison of long-term outcome between anti-Jo1- and anti-PL7/PL12 positive patients with antisynthetase syndrome. Autoimmunity reviews 2012, 11(10):739-745.

44. Marie I, Hatron PY, Dominique S, Et.al: Short-term and long-term outcomes of interstitial lung disease in polymyositis and dermatomyositis: a series of 107 patients. Arthritis and rheumatism 2011, 63(11):3439-3447.

45. Aggarwal R, Cassidy E, Fertig N, et.al: Patients with non-Jo-1 anti-tRNA-synthetase autoantibodies have worse survival than Jo-1 positive patients. Annals of the rheumatic diseases 2014, 73(1):227-232.

46. Marie I, Hatron PY, Dominique S, Et.al: Short-term and long-term outcome of anti-Jo1positive patients with anti-Ro52 antibody. Seminars in arthritis and rheumatism 2012, 41(6):890-899.

47. Marie I, Josse S, Decaux O, Et.al: Clinical manifestations and outcome of anti-PL7 positive patients with antisynthetase syndrome. European journal of internal medicine 2013, 24(5):474-479.

48. Mulcahy KP, Langdon PC, Mastaglia F: Dysphagia in inflammatory myopathy: self-report, incidence, and prevalence. Dysphagia 2012, 27(1):64-69. 
Table.1. Demography and mortality rates in the Polymyositis (PM), Dermatomyositis (DM) and Inclusion Body Myositis (sIBM) patient study cohorts

\begin{tabular}{|c|c|c|c|}
\hline \multicolumn{4}{|l|}{ Demography } \\
\hline & $\mathbf{P M}$ & DM & sIBM \\
\hline Patients $(\mathrm{N})$ & 98 & 128 & 100 \\
\hline Female/male ratio & $1.6: 1$ & $2.1: 1$ & $0.7: 1$ \\
\hline Age at diagnosis yrs. Mean (SD) & $55.6(1.5)$ & $51.4(1.4)$ & $66.9(0.9)$ \\
\hline Disease duration yrs. Mean (SD) & $8.9(0.8)$ & $7.0(0.7)$ & $5.5(0.5)$ \\
\hline Diagnostic delay yrs. Mean (SD) & $1.6(0.3)$ & $1.4(0.4)$ & $5.6(0.5)$ \\
\hline Deceased Number & 30 & 26 & 31 \\
\hline Mean time to death yrs. (CI 95\%). & $5.5(3.1,7.9)$ & $4.2(1.7,6.7)$ & $6.5(4.6,8.4)$ \\
\hline Anti-Jo-1 positive, n/N (\%) & $18 / 82(22.0 \%)$ & $47 / 120(39.2 \%)$ & $1 / 65(1.5 \%)$ \\
\hline MAA positive & $41 / 81(50.6 \%)$ & $71 / 120(59.2 \%)$ & $6 / 59(10.2 \%)$ \\
\hline \multicolumn{4}{|l|}{ Mortality rates (CI $95 \%$ ) } \\
\hline $\mathrm{SMR}^{*}$ & $2.4(1.7,3.3)$ & $2.6(1.8,3.9)$ & $1.7(1.2,2.3)$ \\
\hline SMR, diagnosis before 2003 & $1.9(1.1,3.2)$ & $1.3(0.5,3.5)$ & $1.4(1.0,2.1)$ \\
\hline SMR, diagnosis 2003-2012 & $2.9(1.9,4.4)$ & $3.2(2.1,4.9)$ & $1.9(1.2,2.9)$ \\
\hline
\end{tabular}

*Standardized Mortality rate was calculated by using 15 age- and gender matched controls pr. Patient

** MAA; myositis associated antibodies, i.e. Jo-1,PL7, 
Table 2: Causes of death, divided into disease related and disease-unrelated, in PM, DM and sIBM patients.

\begin{tabular}{|c|c|c|c|c|}
\hline & PM & DM & sIBM & Total \\
$(\mathrm{N}=98)$ & $(\mathrm{N}=128)$ & $(\mathrm{N}=100)$ & $(\mathrm{N}=326)$ \\
\hline Total deaths & 30 & 26 & 31 & 87 \\
\hline Disease-related causes of death (68\%) & 19 & 21 & 16 & 56 \\
\hline ILD/PH* & 5 & 9 & 0 & 14 \\
\hline Infection & 4 & 2 & 4 & 10 \\
\hline Cancer & 5 & 9 & 3 & 17 \\
\hline Aspiration and dysphagia & 3 & 0 & 7 & 10 \\
\hline Falls and fatal injuries & 2 & 1 & 2 & 5 \\
\hline Causes of death, disease-unrelated (32\%) & 11 & 5 & 15 & 31 \\
\hline Heart failure and infarction & 3 & 1 & 8 & 12 \\
\hline Cancer & 3 & 4 & 0 & 7 \\
\hline Miscellaneous** & 3 & 0 & 3 & 6 \\
\hline Unknown cause & 2 & 0 & 4 & 6 \\
\hline
\end{tabular}

*ILD=Interstitial Lung Disease and $\mathrm{PH}=$ Pulmonary Hypertension

**Miscellaneous: Cerebral bleeds and infarction, Diabetes Mellitus, general atherosclerosis, liver cirrhosis, portal hypertension, oesophageal varices. 
Table 3: Assessment of mortality risk factors in PM, DM and sIBM patients. Risk expressed as Hazard Ratios with 95\% confidence intervals and P-values. Risk factors with significance level $\mathrm{P}<0.05$ are expressed in bold types.

\begin{tabular}{|c|c|c|c|}
\hline Risk factor & PM & DM & sIBM \\
\hline Gender & $\begin{array}{c}1.3(0.6,2.8) \\
\mathrm{P}=0.49\end{array}$ & $\begin{array}{c}0.8(0.3,1.9) \\
\mathrm{P}=0.55\end{array}$ & $\begin{array}{c}0.9(0.5,1.9) \\
\mathrm{P}=0.84\end{array}$ \\
\hline Age at diagnosis & $\begin{array}{c}1,1(1.1,1.1) \\
P=0.00\end{array}$ & $\begin{array}{c}1.1(1.0,1.1) \\
P=00\end{array}$ & $\begin{array}{c}1.2(1.1,1.2) \\
P=00\end{array}$ \\
\hline Disease duration & $\begin{array}{c}0.3(0.2,0.5) \\
P=0.00\end{array}$ & $\begin{array}{c}0.5(0.3,0.7) \\
P=0.00\end{array}$ & $\begin{array}{c}0.5(0.4,0.7) \\
P=0.00\end{array}$ \\
\hline Diagnostic delay & $\begin{array}{c}0.8(0.7,1.2) \\
P=0.15\end{array}$ & $\begin{array}{c}1.0(0.9,1.1) \\
\mathrm{P}=0.81\end{array}$ & $\begin{array}{c}1.0(0.9,1.1) \\
\mathrm{P}=0-51\end{array}$ \\
\hline Oesophagus dysmotility & $\begin{array}{c}0.7(0.3,1.5) \\
\mathrm{P}=0.36\end{array}$ & $\begin{array}{c}0.7(0.3,1.7) \\
\mathrm{P}=0.43\end{array}$ & $\begin{array}{c}0.6(0.3,1.2) \\
\mathrm{P}=0.14\end{array}$ \\
\hline $\mathrm{DLCO}<60 \%$ & $\begin{array}{c}12.9(2.9,58.1) \\
P=0.00\end{array}$ & $\begin{array}{c}4.9(1.3,18.2) \\
P=0.02\end{array}$ & $\begin{array}{c}1.1(0.1,9.9) \\
\mathrm{P}=0.93\end{array}$ \\
\hline $\mathrm{FVC}<70 \%$ & $\begin{array}{c}1.5(0.5,4,0) \\
\mathrm{P}=0.54\end{array}$ & $\begin{array}{c}6.8(1.8,25.6) \\
P=0.00\end{array}$ & $\begin{array}{c}1.7(0.3,9.0) \\
\mathrm{P}=0.52\end{array}$ \\
\hline MAA & $\begin{array}{c}1.2(0.7,2.0) \\
\mathrm{P}=0.54\end{array}$ & $\begin{array}{c}1.2(0.6,2.2) \\
\mathrm{P}=0.57\end{array}$ & $\begin{array}{c}1.2(0.6,2.3) \\
\mathrm{P}=0.56\end{array}$ \\
\hline Anti-Jo1 & $\begin{array}{c}1.0(0.5,1.8) \\
\mathrm{P}=0.86\end{array}$ & $\begin{array}{c}0.8(0.4,1.7) \\
\mathrm{P}=0.59\end{array}$ & $\begin{array}{c}1.7(0.8,3.5) \\
\mathrm{P}=0.18\end{array}$ \\
\hline Anti-SSA & $\begin{array}{c}1.0(0.6,1.7) \\
\mathrm{P}=0.99\end{array}$ & $\begin{array}{c}1.3(0.7,2.3) \\
\mathrm{P}=0.49\end{array}$ & $\begin{array}{c}1.4(0.8,2.5) \\
\mathrm{P}=0.22\end{array}$ \\
\hline Cancer & $\begin{array}{c}2.7(1.2,6.0) \\
P=0.01\end{array}$ & $\begin{array}{c}9.2(3.9,21.9) \\
P=0.00\end{array}$ & $\begin{array}{c}2.5(1.1,5.6) \\
P=0,03\end{array}$ \\
\hline
\end{tabular}




\section{FIGURE LEGENDS}

Figure 1 (A) Survival functions measured by survival rates at 2.5 and 10 years shown in percentage of PM patients and matched controls depictured in column diagrams. (B) and. (C) estimated survival rates in dermatomyositis (DM) and sporadic Inclusion Body Myositis (sIBM), respectively.

Figure2 Diagrams showing time from diagnosis (10 years prior to and 10 years after IIM diagnosis) to cancer debut in PM, DM and sIBM patients. Number of cancers seen in each disease group is shown on the vertical line. PM is depictured as blue, DM as red and sIBM as green. 
A.

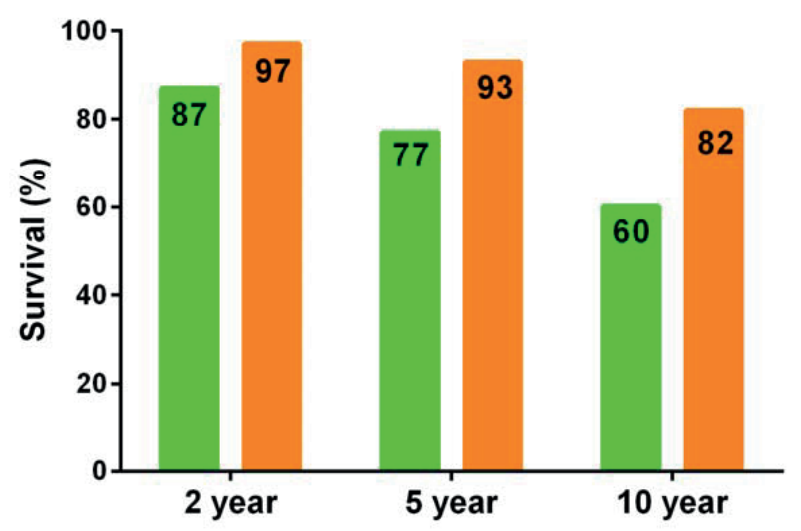

B.

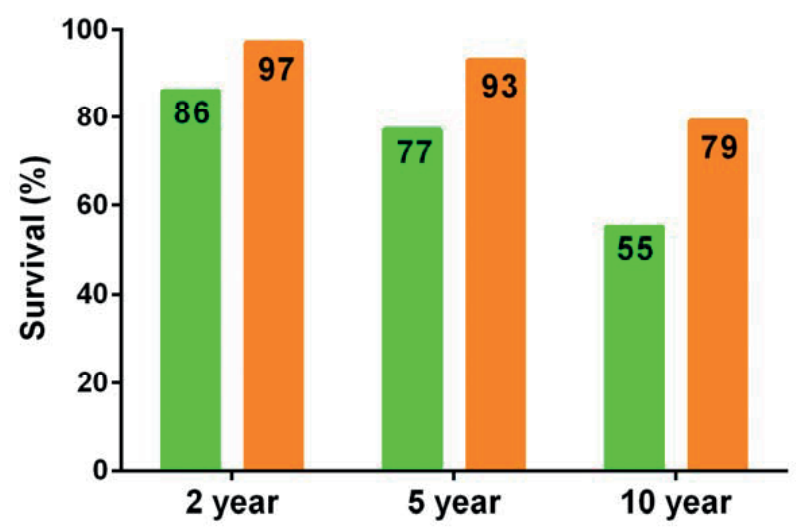

C.

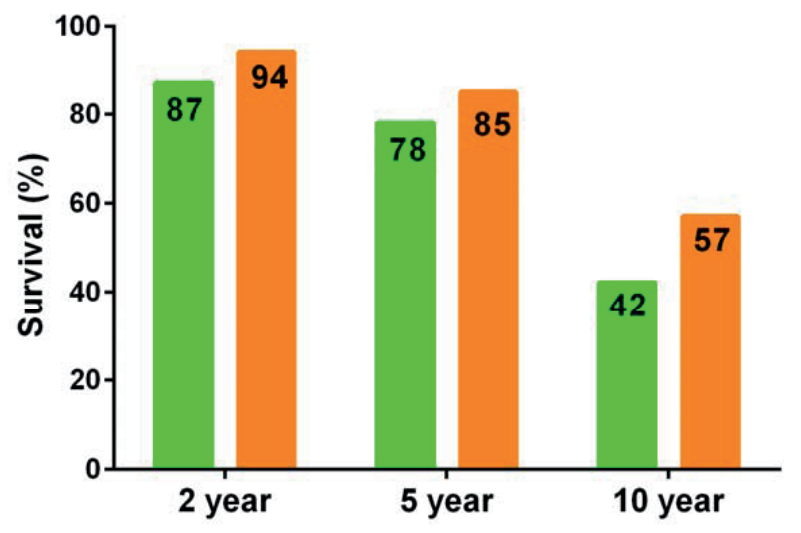




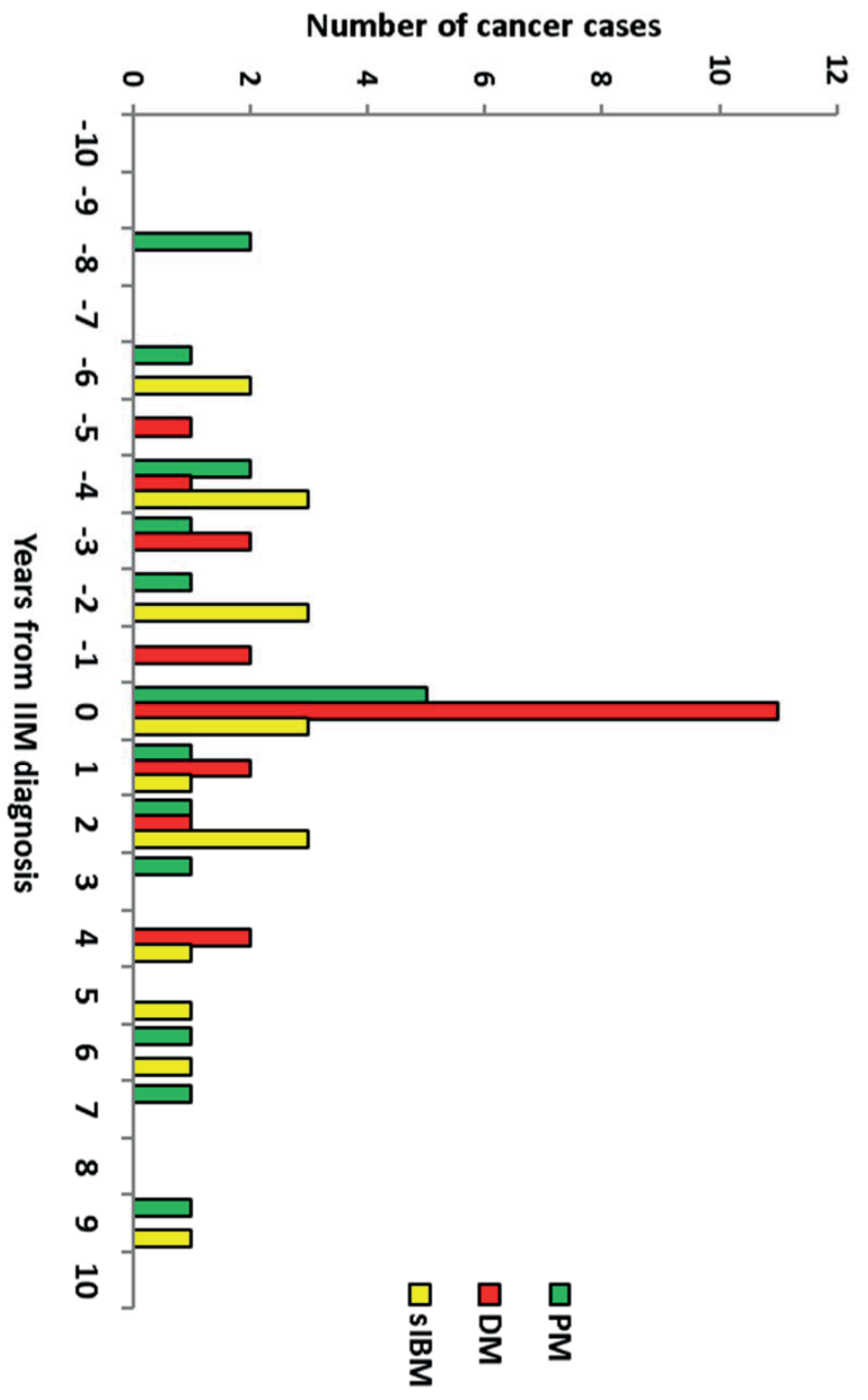

